\title{
CONVERGENCE OF A PARAMETRIC CONTINUATION METHOD
}

\author{
Maroju Prashanth and Dharmendra K. Gupta ${ }^{\dagger}$
}

\begin{abstract}
The aim of this paper is to establish the semilocal convergence of a parameter based continuation method combining the Chebyshev's and the Super-Halley's methods for solving nonlinear equations in Banach spaces. The parameter $\alpha \in[0,1]$ be such that for $\alpha=0$ it reduces to the Chebyshev's method and for $\alpha=1$ to the Super-Halley's method. This convergence is established using recurrence relations under the assumption that the second order Fréchet derivative satisfies the $\omega$-continuity condition. This condition is milder than the Lipschitz and the Hölder continuity conditions used for this purpose. A numerical example is given to show that the second order Fréchet derivative satisfies the $\omega$-continuity condition even when it fails to satisfy the Lipschitz and the Hölder continuity conditions. A number of recurrence relations are derived based on two parameters. The existence and uniqueness regions along with a closed form of the error bounds in terms of a real parameter $\alpha \in[0,1]$ for the solution $x^{*}$ is given. Two numerical examples are worked out to demonstrate the efficacy of the method. It is observed that our method gives better existence and uniqueness regions of the solution for both the examples when compared with the results obtained in [4] for both the Chebyshev's method $(\alpha=0)$ and the Super-Halley's method $(\alpha=1)$.
\end{abstract}

\section{Introduction}

Let $X$ and $Y$ be (real or complex) Banach spaces, $\Omega \subseteq X$ an open subset and let $F: \Omega \subseteq X \rightarrow Y$ be a nonlinear operator with a second order Fréchet derivative on $\Omega$. The problem of approximating a solution $x^{*}$ of nonlinear equation

$$
F(x)=0
$$

is one of the most interesting problems in numerical analysis and scientific computing. With the development of fast, reliable and efficient computers, this problem has further gained an added importance. There exists a large number of applications that give rise to thousands of such equations depending on one or more parameters. For example, dynamical systems are mathematically modeled

Mathematics Subject Classification. 47H10, 41A25, 65Q05.

Key words and phrases. Nonlinear operator equations, $\omega$-continuity condition, Recurrence relations, $R$-order of convergence, A priori error bounds.

$\dagger$ Corresponding author.

Received March 6, 2013; revised September 17, 2013. 
by difference or differential equations and their solutions usually represent the equilibrium states of the systems obtained by solving nonlinear equations. Newton's method is the well known quadratical convergent iterative method for solving (1). But, recently a lot of research has been carried out to provide improvements in these methods. As a result, many iterative methods along with their local, semilocal and global convergence analysis are discussed in [12, 15]. The semilocal convergence analysis use conditions on $F$ and the initial approximation $x_{0}$. If we use conditions only on $F$ then the convergence analysis is global. The local convergence analysis uses conditions only on $x^{*}$. The convergence analysis is done either by using majorizing sequences or recurrence relations under various continuity conditions on the first/second order Fréchet derivatives of the involved operator. The well known third order iterative methods for solving (1) are the Halley's, the Chebyshev's and the Super-Halley's methods. These methods are useful in solving stiff systems of equations, where a quick convergence is required. The main difficulties in these third order iterative methods is to evaluate the second order Fréchet derivative of the operator $F$. In fact, a very restrictive condition of one point iteration of order $N$ is that they depend explicitly on the first $N-1$ derivatives of $F$. This implies that their informational efficiency is less than or equal to unity. Candela and Marquina [2] discussed the semilocal convergence of the Chebyshev's method using recurrence relations under the assumption that the second order Fréchet derivative satisfies the Lipschitz continuity condition. Hernández and Salanova [9] studied the semilocal convergence of the Chebyshev's method under the assumption that the second order Fréchet derivative satisfies the Hölder continuity condition. The semilocal convergence analysis of Super-Halley's method with second order Fréchet derivative satisfying the Lipschitz continuity condition using recurrence relations is described in [7]. Prashanth and Gupta [14] established the semilocal convergence of the Super-Halley's method using recurrence relations under the assumption that the second order Fréchet derivative satisfies the Hölder continuity condition. Xintao and Chong [16] discussed the family of deformed EulerHalley iterations with second order Fréchet derivative satisfying the Hölder condition. However, the Lipschtiz and Hölder continuity conditions on $F^{\prime \prime}$ may be violated by many problems. $[5]:$

Example. Consider the following nonlinear integral equation of mixed type

$$
F(x)(s)=x(s)+\sum_{i=1}^{m} \int_{a}^{b} k_{i}(s, t) l_{i}(x(t)) d t-u(s), \quad s \in[a, b]
$$

where $-\infty<a<b<\infty, u, l_{i}$, and $k_{i}$, for $i=1,2, \ldots, m$ are known functions and $x$ is a continuous function.

If $l_{i}^{\prime \prime}(x(t))$ is $L_{i}$-Lipschitz continuous in $\Omega, L_{i} \geq 0$, for $i=1,2, \ldots, m$, then $F^{\prime \prime}$ does not satisfy Lipschitz condition, where sup-norm is considered. In this 
case

$$
\left\|F^{\prime \prime}(x)-F^{\prime \prime}(y)\right\|=\sum_{i=1}^{m} L_{i}\|x-y\|, \quad x, y \in \Omega .
$$

Similarly, if $l_{i}^{\prime \prime}(x(t))$ is $\left(L_{i}, p_{i}\right)$-Hölder continuous in $\Omega, L_{i} \geq 0, p_{i} \in(0,1]$ for $i=1,2, \ldots, m$, then we have

$$
\left\|F^{\prime \prime}(x)-F^{\prime \prime}(y)\right\|=\sum_{i=1}^{m} L_{i}\|x-y\|^{p_{i}}, \quad x, y \in \Omega .
$$

Here also, $F^{\prime \prime}$ is not Hölder continuous, when sup-norm is used.

A continuation method is a parameter based method giving a continuous connection between two functions $f$ and $g$. Mathematically, a continuation method between two functions $f, g: X \rightarrow Y$, where $X$ and $Y$ are Banach spaces is defined as a continuous map $h:[0,1] \times X \rightarrow Y$ such that $h(\alpha, x)=\alpha f(x)+$ $(1-\alpha) g(x), \quad \alpha \in[0,1]$ and $h(0, x)=g(x), h(1, x)=f(x)$. The continuation method was known as early as 1930s. It was used by Kinemitician in the 1960s for solving mechanism synthesis problems. It also gives a set of certain answers and a simple iteration process to obtain solutions more exactly. For further literature survey on it, one can refer to the works of $[1,11]$. Recently, Gupta and Prashanth [6] discussed the semilocal convergence analysis of a continuation method using recurrence relations under the assumption that the second order Fréchet derivative satisfies the Hölder continuity condition. Ezquerro and Hernández [4] have considered the $\omega$-continuity condition on $F^{\prime \prime}$ given by

$$
\left\|F^{\prime \prime}(x)-F^{\prime \prime}(y)\right\| \leq \omega(\|x-y\|), \quad x, y \in \Omega,
$$

where, $\omega(x)$ is a nondecreasing continuous real function for $x>0$, such that $\omega(0) \geq 0$, to study the semilocal convergence of a family of third order iterative method. Parida and Gupta [13] established the convergence analysis using recurrence relations of the Chebyshev method under the $\omega$-continuity condition on $F^{\prime \prime}$. The semilocal convergence using recurrence relations of the Super-Halley's method under the $\omega$-continuity condition on $F^{\prime \prime}$ is also established by them.

The aim of this paper is to establish the semilocal convergence of a parameter based continuation method combining the Chebyshev's and the SuperHalley's methods for solving nonlinear equations in Banach spaces. The parameter $(\alpha \in[0,1])$ be such that for $\alpha=0$ it reduces to the Chebyshev's method and for $\alpha=1$ to the Super-Halley's method. This convergence is established using recurrence relations under the assumption that the second order Fréchet derivative satisfies the $\omega$-continuity condition. This condition is milder than the Lipschitz and the Hölder continuity conditions used for this purpose. A numerical example is given to show that the second order Fréchet derivative satisfies the $\omega$-continuity condition even when it fails to satisfy the Lipschitz and the Hölder continuity conditions. A number of recurrence relations are derived based on two parameters. The existence and uniqueness regions along with a 
closed form of the error bounds in terms of a real parameter $\alpha \in[0,1]$ for the solution $x^{*}$ is given. Two numerical examples are worked out to demonstrate the efficacy of the method. It is observed that our method gives better existence and uniqueness regions of the solution for both the examples when compared with the results obtained in [4] for both the Chebyshev's method $(\alpha=0)$ and the Super-Halley's method $(\alpha=1)$.

This paper is organized in five Sections. Section 1 is the introduction. In Section 2, the continuation method combining the Chebyshev's method and the Super-Halley's method to solve nonlinear equations in Banach spaces and a number of recurrence relations based on two parameters are derived. The convergence analysis for the continuation method under the assumption that the second Fréchet derivative satisfies the $\omega$-continuity condition is established in Section 3. An existence and uniqueness theorem along with the estimation of a priori error bounds in terms of a real parameter $\alpha \in[0,1]$ is provided. The $R$-order of the method is also analyzed. In Section 4, two numerical examples are worked out to demonstrate the efficacy of our convergence analysis. Finally, conclusions form the Section 5 .

\section{Recurrence relations}

In this section, we shall first describe the continuation method combining the Chebyshev's method and the Super-Halley's method to solve (1) and then derive a family of recurrence relations based on two parameters. Let $F^{\prime}\left(x_{0}\right)^{-1} \in$ $B L(Y, X)$ exist at some point $x_{0} \in \Omega$, where $B L(Y, X)$ be the set of bounded linear operators from $Y$ into $X$. The Chebyshev's method and the SuperHalley's method used for solving (1) are defined for $n=0,1,2, \ldots$ by

$$
x_{n+1}=J_{0}\left(x_{n}\right)=x_{n}-\left[I+\frac{1}{2} L_{F}\left(x_{n}\right)\right] F^{\prime}\left(x_{n}\right)^{-1} F\left(x_{n}\right)
$$

and

$$
x_{n+1}=J_{1}\left(x_{n}\right)=x_{n}-\left[I+\frac{1}{2} L_{F}\left(x_{n}\right)\left(I-L_{F}\left(x_{n}\right)\right)^{-1}\right] F^{\prime}\left(x_{n}\right)^{-1} F\left(x_{n}\right)
$$

where, $I$ is identity operator and $L_{F}\left(x_{n}\right)$ is the linear operator given by

$$
L_{F}\left(x_{n}\right)=F^{\prime}\left(x_{n}\right)^{-1} F^{\prime \prime}\left(x_{n}\right) F^{\prime}\left(x_{n}\right)^{-1} F\left(x_{n}\right), \quad x_{n} \in X .
$$

If $F^{\prime}\left(x_{\alpha, 0}\right)^{-1} \in B L(Y, X)$ for $x_{\alpha, 0} \in \Omega, \alpha \in[0,1]$, then the continuation method between (3) and (4) can be defined by

$$
x_{\alpha, n+1}=\alpha J_{1}\left(x_{\alpha, n}\right)+(1-\alpha) J_{0}\left(x_{\alpha, n}\right) \quad n \geq 0 .
$$

Replacing $x_{n}$ by $x_{\alpha, n}$ in (3), (4) and (5) and substituting expressions for $J_{0}\left(x_{\alpha, n}\right)$ and $J_{1}\left(x_{\alpha, n}\right)$ into $(6)$, we get

$$
x_{\alpha, n+1}=x_{\alpha, n}-\left[I+\frac{1}{2} L_{F}\left(x_{\alpha, n}\right) G_{\alpha}\left(x_{\alpha, n}\right)\right] F^{\prime}\left(x_{\alpha, n}\right)^{-1} F\left(x_{\alpha, n}\right)
$$


where,

$$
\begin{gathered}
G_{\alpha}\left(x_{\alpha, n}\right)=I+\alpha L_{F}\left(x_{\alpha, n}\right) H_{\alpha}\left(x_{\alpha, n}\right) \\
H_{\alpha}\left(x_{\alpha, n}\right)=\left(I-L_{F}\left(x_{\alpha, n}\right)\right)^{-1}
\end{gathered}
$$

and

$$
L_{F}\left(x_{\alpha, n}\right)=F^{\prime}\left(x_{\alpha, n}\right)^{-1} F^{\prime \prime}\left(x_{\alpha, n}\right) F^{\prime}\left(x_{\alpha, n}\right)^{-1} F\left(x_{\alpha, n}\right) .
$$

Simplifying (7), the continuation method can be given as follows. Starting with a suitably chosen $x_{\alpha, 0}$, define

$$
\left.\begin{array}{l}
y_{\alpha, n}=x_{\alpha, n}-F^{\prime}\left(x_{\alpha, n}\right)^{-1} F\left(x_{\alpha, n}\right) \\
x_{\alpha, n+1}=y_{\alpha, n}+\frac{1}{2} L_{F}\left(x_{\alpha, n}\right) G_{\alpha}\left(x_{\alpha, n}\right)\left(y_{\alpha, n}-x_{\alpha, n}\right)
\end{array}\right\}
$$

for $n=0,1,2, \ldots$ and $G_{\alpha}\left(x_{\alpha, n}\right)$ given by (8). Let the following assumptions hold.

C1. $\left\|F^{\prime}\left(x_{\alpha, 0}\right)^{-1}\right\| \leq \beta$,

C2. $\left\|F^{\prime}\left(x_{\alpha, 0}\right)^{-1} F\left(x_{\alpha, 0}\right)\right\| \leq \eta$,

C3. $\left\|F^{\prime \prime}(x)\right\| \leq M, \quad \forall x \in \Omega$,

C4. $\left\|F^{\prime \prime}(x)-F^{\prime \prime}(y)\right\| \leq \omega(\|x-y\|), \forall x, y \in \Omega$, where $\omega: \mathbf{R}_{+} \rightarrow \mathbf{R}_{+}$ is a continuous and nondecreasing function such that $\omega(0) \geq 0$,

C5. There exists a continuous and nondecreasing function $h:[0,1] \rightarrow \mathbf{R}_{+}$such that, $\omega(t x) \leq h(t) \omega(x)$, with $t \in[0,1]$ and $x \in \mathbf{R}_{+}$.

The condition $\mathrm{C} 4$ of $(10)$ is milder than the Lipschitz/Hölder continuity condition as this condition reduces to the Lipschitz and the Hölder continuity conditions for $\omega(x)=N x$ and $\omega(x)=N x^{p}, p \in(0,1]$, respectively. Also, note that the condition $\mathrm{C} 5$ of (10) does not involve any restriction, since, as a consequence of $\omega$ be a nondecreasing function, there always exists a function $h$ such that $h(t)=1$. We can consider $h(t)=\sup _{x>0} \omega(t x) / \omega(x)$ to sharpen the error bounds for a particular case. For $n=0,1,2 \ldots$, define the real sequences $\left\{a_{n}\right\},\left\{b_{n}\right\}$ and $\left\{c_{n}\right\}$ for

$$
c_{n}=f\left(a_{n}\right) g\left(a_{n}, b_{n}\right), \quad a_{n+1}=a_{n} f\left(a_{n}\right) c_{n}, \quad b_{n+1}=b_{n} f\left(a_{n}\right) c_{n} h\left(c_{n}\right)
$$

where,

$$
\begin{gathered}
f(x)=\frac{2(1-x)}{2-4 x+x^{2}-(\alpha-1) x^{3}} \\
g(x, y)=\frac{\alpha x^{2}}{2(1-x)}+\frac{A y(1+(\alpha-1) x)}{(1-x)} \\
+\frac{x^{2}(1+(\alpha-1) x)}{2(1-x)}+\frac{x^{3}(1+(\alpha-1) x)^{2}}{8(1-x)^{2}}
\end{gathered}
$$


for $A$ defined by

$$
A=\int_{0}^{1} h(t)(1-t) d t
$$

Let $a_{0}=M \beta \eta$ and $b_{0}=\beta \eta \omega(\eta)$ are two parameters and $y_{\alpha, 0} \in \Omega$ exists since $\Gamma_{\alpha, 0}=F^{\prime}\left(x_{\alpha, 0}\right)^{-1}$ exists. For $y_{\alpha, 0} \in \Omega$, we get

$$
\left\|L_{F}\left(x_{\alpha, 0}\right)\right\| \leq M\left\|\Gamma_{\alpha, 0}\right\|\left\|\Gamma_{\alpha, 0} F\left(x_{\alpha, 0}\right)\right\| \leq M \beta \eta=a_{0}<1
$$

By Banach Lemma, we get

$$
\left\|H_{\alpha}\left(x_{\alpha, 0}\right)\right\| \leq \frac{1}{1-a_{0}}
$$

and

$$
\left\|G_{\alpha}\left(x_{\alpha, 0}\right)\right\| \leq \frac{1+(\alpha-1) a_{0}}{1-a_{0}}
$$

This gives

$$
\left\|x_{\alpha, 1}-x_{\alpha, 0}\right\| \leq \frac{\left(2-a_{0}+(\alpha-1) a_{0}^{2}\right)}{2\left(1-a_{0}\right)}\left\|y_{\alpha, 0}-x_{\alpha, 0}\right\|
$$

and

$$
\left\|\Gamma_{\alpha, 0}\right\|\left\|y_{\alpha, 0}-x_{\alpha, 0}\right\| \omega\left(\left\|y_{\alpha, 0}-x_{\alpha, 0}\right\|\right) \leq \beta \eta \omega(\eta) .
$$

Under the assumptions (10), the following inequalities hold for $n \geq 1$.

(I) $\left\|\Gamma_{\alpha, n}\right\|=\left\|F^{\prime}\left(x_{\alpha, n}\right)^{-1}\right\| \leq f\left(a_{n-1}\right)\left\|\Gamma_{\alpha, n-1}\right\|$,

(II) $\left\|y_{\alpha, n}-x_{\alpha, n}\right\|=\left\|\Gamma_{\alpha, n} F\left(x_{\alpha, n}\right)\right\| \leq f\left(a_{n-1}\right) g\left(a_{n-1}, b_{n-1}\right)\left\|\Gamma_{\alpha, n} F\left(x_{\alpha, n}\right)\right\|$,

(III) $\left\|L_{F}\left(x_{\alpha, n}\right)\right\| \leq M\left\|\Gamma_{\alpha, n}\right\|\left\|y_{\alpha, n}-x_{\alpha, n}\right\| \leq a_{n}$,

(IV) $\left\|\Gamma_{\alpha, n}\right\|\left\|y_{\alpha, n}-x_{\alpha, n}\right\| \omega\left(\left\|y_{\alpha, n}-x_{\alpha, n}\right\|\right) \leq b_{n}$

(V) $\left\|x_{\alpha, n+1}-x_{\alpha, n}\right\| \leq \frac{\left(2-a_{n}+(\alpha-1) a_{n}^{2}\right)}{2\left(1-a_{n}\right)}\left\|y_{\alpha, n}-x_{\alpha, n}\right\|$.

We shall use mathematical induction to prove $(\mathrm{I})-(\mathrm{V})$. Assume that $x_{\alpha, 1} \in \Omega$ and $0<a_{0}<r_{0}$. From

$$
\left\|I-\Gamma_{\alpha, 0} F^{\prime}\left(x_{\alpha, 1}\right)\right\| \leq M\left\|\mid \Gamma_{\alpha, 0}\right\|\left\|x_{\alpha, 0}-x_{\alpha, 1}\right\| \leq \frac{a_{0}\left(2-a_{0}+(\alpha-1) a_{0}^{2}\right)}{2\left(1-a_{0}\right)}<1 .
$$

Using Banach Lemma, $\Gamma_{\alpha, 1}=F^{\prime}\left(x_{\alpha, 1}\right)^{-1}$ exists and

$$
\begin{aligned}
\left\|\Gamma_{\alpha, 1}\right\| & \leq \frac{\left\|\Gamma_{\alpha, 0}\right\|}{1-M\left\|\Gamma_{\alpha, 0}\right\|\left\|x_{\alpha, 0}-x_{\alpha, 1}\right\|} \\
& \leq \frac{2\left(1-a_{0}\right)}{2-4 a_{0}+a_{0}^{2}-(\alpha-1) a_{0}^{3}}\left\|\Gamma_{\alpha, 0}\right\| \\
& \leq f\left(a_{0}\right)\left\|\Gamma_{\alpha, 0}\right\| .
\end{aligned}
$$


Now, $y_{\alpha, 1}$ exists as $\Gamma_{\alpha, 1}$ exists and

$$
x_{\alpha, n+1}-y_{\alpha, n}=\frac{1}{2} L_{F}\left(x_{\alpha, n}\right) G_{\alpha}\left(x_{\alpha, n}\right)\left(y_{\alpha, n}-x_{\alpha, n}\right) .
$$

Expanding $F\left(x_{\alpha, n+1}\right)$ by using Taylor's theorem, we get

$$
\begin{aligned}
F\left(x_{\alpha, n+1}\right)= & F\left(y_{\alpha, n}\right)+F^{\prime}\left(y_{\alpha, n}\right)\left(x_{\alpha, n+1}-y_{\alpha, n}\right)+\int_{y_{\alpha, n}}^{x_{\alpha, n+1}} F^{\prime \prime}(x)\left(x_{\alpha, n+1}-x\right) d x \\
= & \int_{0}^{1}\left[F^{\prime \prime}\left(x_{\alpha, n}+t\left(y_{\alpha, n}-x_{\alpha, n}\right)\right)-F^{\prime \prime}\left(x_{\alpha, n}\right)\right](1-t) d t G_{\alpha}\left(x_{\alpha, n}\right)\left(y_{\alpha, n}-x_{\alpha, n}\right)^{2} \\
& +\int_{0}^{1} F^{\prime \prime}\left(x_{\alpha, n}+t\left(y_{\alpha, n}-x_{\alpha, n}\right)\right)(1-t) d t\left(I-G_{\alpha}\left(x_{\alpha, n}\right)\right)\left(y_{\alpha, n}-x_{\alpha, n}\right)^{2} \\
& +\int_{0}^{1} F^{\prime \prime}\left(x_{\alpha, n}+t\left(y_{\alpha, n}-x_{\alpha, n}\right)\right) d t\left(y_{\alpha, n}-x_{\alpha, n}\right)\left(x_{\alpha, n+1}-y_{\alpha, n}\right) \\
& +\int_{0}^{1} F^{\prime \prime}\left(y_{\alpha, n}+t\left(x_{\alpha, n+1}-y_{\alpha, n}\right)\right)(1-t) d t\left(x_{\alpha, n+1}-y_{\alpha, n}\right)^{2} .
\end{aligned}
$$

Taking norm on both sides, this gives

$$
\begin{aligned}
\left\|F\left(x_{\alpha, n+1}\right)\right\| \leq & \frac{M}{2}\left\|I-G_{\alpha}\left(x_{\alpha, n}\right)\right\|\left\|\left(y_{\alpha, n}-x_{\alpha, n}\right)\right\|^{2} \\
& +\int_{0}^{1} \omega\left(t\left(y_{\alpha, n}-x_{\alpha, n}\right)\right)(1-t) d t\left\|G_{\alpha}\left(x_{\alpha, n}\right)\right\|\left\|\left(y_{\alpha, n}-x_{\alpha, n}\right)\right\|^{2} \\
& +M\left\|\left(y_{\alpha, n}-x_{\alpha, n}\right)\right\|\left\|x_{\alpha, n+1}-y_{\alpha, n}\right\| \\
& +\frac{M}{2}\left\|\left(x_{\alpha, n+1}-y_{\alpha, n}\right)\right\|^{2} .
\end{aligned}
$$

From (18) for $n=0$, we get

$$
\begin{aligned}
\left\|F\left(x_{\alpha, 1}\right)\right\| \leq & \frac{M \alpha a_{0}}{2\left(1-a_{0}\right)} \eta^{2}+A \omega(\eta) \frac{\left(1+(\alpha-1) a_{0}\right) \eta^{2}}{\left(1-a_{0}\right)} \\
& +\frac{M \eta^{2} a_{0}\left(1+(\alpha-1) a_{0}\right)}{2\left(1-a_{0}\right)}+\frac{M}{8} \frac{\eta^{2} a_{0}^{2}\left(1+(\alpha-1) a_{0}\right)^{2}}{\left(1-a_{0}\right)^{2}}
\end{aligned}
$$

and

$$
\begin{aligned}
\left\|\Gamma_{\alpha, 1} F\left(x_{\alpha, 1}\right)\right\| & \leq\left\|\Gamma_{\alpha, 1}\right\|\left\|F\left(x_{\alpha, 1}\right)\right\| \\
& \leq f\left(a_{0}\right)\left\|\Gamma_{\alpha, 0}\right\|\left\|F\left(x_{\alpha, 1}\right)\right\|
\end{aligned}
$$




$$
\begin{aligned}
& \leq f\left(a_{0}\right)\left[\frac{\alpha a_{0}^{2}}{2\left(1-a_{0}\right)}+\frac{A b_{0}\left(1+(\alpha-1) a_{0}\right)}{\left(1-a_{0}\right)}\right. \\
& \left.\quad+\frac{a_{0}^{2}\left(1+(\alpha-1) a_{0}\right)}{2\left(1-a_{0}\right)}+\frac{a_{0}^{3}\left(1+(\alpha-1) a_{0}\right)^{2}}{8\left(1-a_{0}\right)^{2}}\right] \eta \\
& \leq f\left(a_{0}\right) g\left(a_{0}, b_{0}\right) \eta \leq c_{0} \eta .
\end{aligned}
$$

Also,

$$
\begin{aligned}
\left\|L_{F}\left(x_{\alpha, 1}\right)\right\| & \leq M\left\|\Gamma_{\alpha, 1}\right\|\left\|\Gamma_{\alpha, 1} F\left(x_{\alpha, 1}\right)\right\| \\
& \leq M f\left(a_{0}\right)\left\|\Gamma_{\alpha, 0}\right\| f\left(a_{0}\right) g\left(a_{0}, b_{0}\right)\left\|\Gamma_{\alpha, 0} F\left(x_{\alpha, 0}\right)\right\| \\
& \leq M \beta \eta f\left(a_{0}\right) f\left(a_{0}\right) g\left(a_{0}, b_{0}\right)=a_{0} f\left(a_{0}\right) c_{0}=a_{1}, \\
\left\|\Gamma_{\alpha, 1}\right\|\left\|y_{\alpha, 1}-x_{\alpha, 1}\right\| \omega\left(\left\|y_{\alpha, 1}-x_{\alpha, 1}\right\|\right) & \\
\leq & f\left(a_{0}\right)\left\|\Gamma_{\alpha, 0}\right\| f\left(a_{0}\right) g\left(a_{0}, b_{0}\right) \\
& \left\|\Gamma_{\alpha, 0} F\left(x_{\alpha, 0}\right)\right\| \omega\left(f\left(a_{0}\right) g\left(a_{0}, b_{0}\right)\right)\left\|\Gamma_{\alpha, 0} F\left(x_{\alpha, 0}\right)\right\| \\
\leq & b_{0} f\left(a_{0}\right) c_{0} h\left(c_{0}\right)=b_{1},
\end{aligned}
$$

and

$$
\left\|G_{\alpha}\left(x_{\alpha, 1}\right)\right\| \leq \frac{\left(1+(\alpha-1) a_{1}\right)}{\left(1-a_{1}\right)} .
$$

Now, from (23) we get

$$
\left\|x_{\alpha, 2}-x_{\alpha, 1}\right\| \leq\left[\frac{\left.2-a_{1}+(\alpha-1) a_{1}^{2}\right)}{2\left(1-a_{1}\right)}\right]\left\|\Gamma_{\alpha, 1} F\left(x_{\alpha, 1}\right)\right\| .
$$

Using (17) and (20) to (24), the inequalities (I)-(V) hold for $n=1$. Assume that they hold for some $n=k$. Proceeding similarly as above, we easily prove that the conditions $(\mathrm{I})-(\mathrm{V})$ also hold for $n=k+1$. Hence by induction they hold for all $n \geq 1$.

\section{Convergence analysis}

In this section, the convergence analysis of the continuation method (9) is established. Let $r_{0}=0.380778$ be the smallest positive zero of the polynomial given by $d(x)=2(\alpha-1) x^{6}-\left(\alpha^{2}+2 \alpha-3\right) x^{5}+2(9 \alpha-8) x^{4}-(8 \alpha+1) x^{3}-$ $(4 \alpha-9) x^{2}-32 x+8, \alpha \in[0,1]$. The following Lemmas will be used to prove the convergence theorem.

Lemma 1. Let $\alpha \in[0,1]$ and the real functions $f$ and $g$ be given by (12) and (13) respectively. Then, for $x \in\left(0, r_{0}\right)$, 
(i) $f$ is a increasing function and $f(x)>1$ in $\left(0, r_{0}\right]$.

(ii) $g$ is a increasing in both arguments for $y>0$.

(iii) $f(\gamma x)<f(x)$ and $g(\gamma x, \gamma y) \leq \gamma g(x, y)$.

Proof. The proof is simple and hence omitted here. Define

LEMMA 2. Let $f$ and $g$ given by (12) and (13) and $h(t) \leq 1 \quad \forall t \in[0,1]$.

$\Phi(x)=\frac{2(\alpha-1) x^{6}-\left(\alpha^{2}+2 \alpha-3\right) x^{5}+2(9 \alpha-8) x^{4}-(8 \alpha+1) x^{3}-(4 \alpha-9) x^{2}-32 x+8}{8 A(1+(\alpha-1) x)(1-x)}$

If $a_{0} \in\left(0, r_{0}\right]$ and $0 \leq b_{0} \leq \Phi\left(a_{0}\right)$, then

(i) $c_{n} f\left(a_{n}\right) \leq 1$,

(ii) $\left\{a_{n}\right\},\left\{b_{n}\right\}$, and $\left\{c_{n}\right\}$ are decreasing and $a_{n}<1, c_{n}<1 \forall n$.

Proof. This Lemma can be proved by induction. From $f$ and $g$, we get

$$
c_{n} f\left(a_{n}\right)=f\left(a_{n}\right)^{2} g\left(a_{n}, b_{n}\right) \leq 1
$$

iff

$$
\begin{gathered}
{\left[\frac{4\left(1-a_{n}\right)^{2}}{\left(2-4 a_{n}+a_{n}^{2}-(\alpha-1) a_{n}^{3}\right)^{2}}\right] \times\left[\frac{\alpha a_{n}^{2}}{2\left(1-a_{n}\right)}+\frac{A b_{n}\left(1+(\alpha-1) a_{n}\right)}{\left(1-a_{n}\right)}\right.} \\
\left.+\frac{a_{n}^{2}\left(1+(\alpha-1) a_{n}\right)}{2\left(1-a_{n}\right)}+\frac{a_{n}^{3}\left(1+(\alpha-1) a_{n}\right)^{2}}{8\left(1-a_{n}\right)^{2}}\right] \leq 1
\end{gathered}
$$

or,

$b_{n} \leq \frac{2(\alpha-1)^{2} a_{n}^{6}-\left(\alpha^{2}+2 \alpha-3\right) a_{n}^{5}+2(9 \alpha-8) a_{n}^{4}-(8 \alpha+1) a_{n}^{3}-(4 \alpha-9) a_{n}^{2}-32 a_{n}+8}{8 A\left(1+(\alpha-1) a_{n}\right)\left(1-a_{n}\right)}$

or,

$$
b_{n} \leq \Phi\left(a_{n}\right)
$$

Thus, $c_{0} f\left(a_{0}\right) \leq 1$ for $0 \leq a_{0} \leq r_{0}$ and $0 \leq b_{0} \leq \Phi\left(a_{0}\right)$. Using (11), we get

$$
a_{1}=a_{0} f\left(a_{0}\right) c_{0} \leq a_{0}<1
$$

Now, for $f(x)>1$ in $\left(0, r_{0}\right]$ and $h(t) \leq 1$ we get

$$
b_{1}=b_{0} f\left(a_{0}\right) c_{0} h\left(c_{0}\right) \leq b_{0} f\left(a_{0}\right) c_{0} \leq b_{0}
$$

and

$$
c_{1}=f\left(a_{1}\right) g\left(a_{1}, b_{1}\right) \leq f\left(a_{0}\right) g\left(a_{0}, b_{0}\right)=c_{0}<c_{0} f\left(a_{0}\right) \leq 1
$$


Thus, the lemma holds for $n=1$. Assume that (i) and (ii) hold for some $n=k$. Proceeding similarly as above, we can easily prove that (i) and (ii) hold for $n=k+1$.

Lemma 3. Let $a_{0} \in\left(0, r_{0}\right), \quad 0<b_{0}<\Phi\left(a_{0}\right)$, and $h(t) \leq 1 \quad \forall t \in[0,1]$. If $\gamma=a_{1} / a_{0}$ then for $n \geq 1$, we have

(i) $a_{n} \leq \gamma^{2^{n-1}} a_{n-1} \leq \gamma^{2^{n}-1} a_{0}$, where, inequality strictly hold for $n \geq 2$,

(ii) $b_{n}<\gamma^{2^{n-1}} b_{n-1}<\gamma^{2^{n}-1} b_{0}$,

(iii) $c_{n}<\gamma^{2^{n}} / f\left(a_{0}\right)$

Proof. The induction will be used to prove (i) and (ii). From $a_{1}=\gamma a_{0}$ and $a_{1}<a_{0}$ from Lemma (ii), we get $\gamma<1$. Hence,

$$
b_{1}=b_{0} f\left(a_{0}\right) c_{0} h\left(c_{0}\right)=b_{0} f\left(a_{0}\right)^{2} g\left(a_{0}, b_{0}\right) h\left(c_{0}\right) \leq f\left(a_{0}\right)^{2} g\left(a_{0}, b_{0}\right) b_{0}=\gamma b_{0}
$$

Thus, (i) and (ii) hold for $n=1$. Assume that (i) and (ii) hold for some $n=k$. Then, by Lemma 1 we get

$$
\begin{aligned}
a_{k+1} & =a_{k} f\left(a_{k}\right) c_{k}=a_{k} f\left(a_{k}\right)^{2} g\left(a_{k}, b_{k}\right) \\
& <\gamma^{2^{k-1}} a_{k-1} f\left(\gamma^{2^{k-1}} a_{k-1}\right)^{2} g\left(\gamma^{2^{k-1}} a_{k-1}, \gamma^{2^{k-1}} b_{k-1}\right) \\
& <\gamma^{2^{k-1}} a_{k-1} f\left(a_{k-1}\right)^{2} \gamma^{2^{k-1}} g\left(a_{k-1}, b_{k-1}\right)=\gamma^{2^{k}} a_{k}
\end{aligned}
$$

Also, from $f(x)>1$ in $\left(0, r_{0}\right)$, we get

$$
b_{k+1}=b_{k} f\left(a_{k}\right) c_{k} h\left(c_{k}\right) \leq b_{k} f\left(a_{k}\right) c_{k}=b_{k} \frac{a_{k+1}}{a_{k}}<\gamma^{2^{k}} b_{k}
$$

This leads to

$$
a_{k+1}<\gamma^{2^{k}} a_{k}<\gamma^{2^{k}} \gamma^{2^{k-1}} \cdots \gamma^{2^{0}} a_{0}=\gamma^{2^{k+1}-1} a_{0}
$$

and

$$
b_{k+1}<\gamma^{2^{k}} b_{k}<\gamma^{2^{k}} \gamma^{2^{k-1}} \cdots \gamma^{2^{0}} b_{0}=\gamma^{2^{k+1}-1} b_{0} .
$$

Hence, (i) and (ii) hold for $n \geq 1$. From $\gamma=a_{1} / a_{0}=f\left(a_{0}\right)^{2} g\left(a_{0}, b_{0}\right)$, we get

$$
\begin{aligned}
c_{n} & =f\left(a_{n}\right) g\left(a_{n}, b_{n}\right)<f\left(\gamma^{2^{n}-1} a_{0}\right) g\left(\gamma^{2^{n}-1} a_{0}, \gamma^{2^{n}-1} b_{0}\right) \\
& <\gamma^{2^{n}-1} f\left(a_{0}\right) g\left(a_{0}, b_{0}\right)=\gamma^{2^{n}} / f\left(a_{0}\right)
\end{aligned}
$$

Hence, (iii) holds. Thus the Lemma 3 is proved.

Let $\quad \gamma=a_{1} / a_{0}, \quad \Delta=1 / f\left(a_{0}\right), \quad R=\frac{2-a_{0}+(\alpha-1) a_{0}^{2}}{2\left(1-a_{0}\right)(1-\gamma \Delta)} \quad$ and $\quad \overline{\mathscr{B}}\left(x_{\alpha, 0}, R \eta\right)=$ $\left\{x \in X ;\left\|x-x_{\alpha, 0}\right\| \leq R \eta\right\} \subseteq \Omega$ and $\mathscr{B}\left(x_{\alpha, 0}, R \eta\right)=\left\{x \in X ;\left\|x-x_{\alpha, 0}\right\|<R \eta\right\} \subseteq \Omega$ are closed and open ball with center $x_{\alpha, 0}$ and radius $R \eta$. 
THEOREM 1. Let $X$ and $Y$ be two Banach spaces and $F: \Omega \subseteq X \rightarrow Y$ be a twice Fréchet differentiable nonlinear operator on a nonempty open convex domain $\Omega$. Let us assume that $\Gamma_{\alpha, 0}$ exist at some $x_{\alpha, 0}$ and the assumptions $(\mathrm{C} 1)-(\mathrm{C} 5)$ are satisfied. Suppose $0<a_{0} \leq r_{0}$ and $0 \leq b_{0} \leq \Phi\left(a_{0}\right)$. Then, the sequence $\left\{x_{\alpha, n}\right\}$ defined in (9) and starting with initial approximation $x_{\alpha, 0}$ has at least, $R$-order two and converges to a solution $x^{*}$ of $F(x)=0$. In that case, the solution $x^{*}$ and the iterates $x_{\alpha, n}$ and $y_{\alpha, n}$ belong to $\overline{\mathscr{B}}\left(x_{\alpha, 0}, R \eta\right)$ and $x^{*}$ is the only solution of $F(x)=0$ in $\mathscr{B}\left(x_{\alpha, 0}, \frac{2}{M \beta}-R \eta\right) \cap \Omega$. Furthermore, a priori error bounds are given by

$$
\left\|x^{*}-x_{\alpha, n}\right\| \leq \frac{2-a_{0} \gamma^{2^{n}-1}+(\alpha-1) \gamma^{2^{n}-1}}{2\left(1-\gamma^{2^{n}-1} a_{0}\right)} \frac{\Delta^{n} \gamma^{2^{n}-1} \eta}{\left(1-\gamma^{2^{n}} \Delta\right)}
$$

Proof. To prove that $\left\{x_{\alpha, n}\right\}$ is convergent, it is sufficient to prove that the sequence $\left\{x_{\alpha, n}\right\}$ is a Cauchy sequence. We have $b_{0}=\Phi\left(a_{0}\right)=0$ and $c_{0} f\left(a_{0}\right)=1$ for $a_{0}=r_{0}$. Hence, from (11), $a_{n}=a_{n-1}=\cdots=a_{0}, c_{n}=c_{n-1}=\cdots=c_{0}$ and $b_{n}=b_{n-1} \cdots=b_{0}=0$. Now, from (16), we have

$$
\begin{aligned}
\left\|y_{\alpha, n}-x_{\alpha, n}\right\| & \leq c_{n-1}\left\|y_{\alpha, n-1}-x_{\alpha, n-1}\right\|=c_{0}\left\|y_{\alpha, n-1}-x_{\alpha, n-1}\right\| \\
& \leq \cdots \leq c_{0}^{n}\left\|y_{\alpha, 0}-x_{\alpha, 0}\right\|=\Delta^{n} \eta
\end{aligned}
$$

and

$$
\left\|x_{\alpha, n+1}-x_{\alpha, n}\right\| \leq \frac{2-a_{n}+(\alpha-1) a_{n}^{2}}{2\left(1-a_{n}\right)}\left\|y_{\alpha, n}-x_{\alpha, n}\right\| \leq \frac{2-a_{0}+(\alpha-1) a_{0}^{2}}{2\left(1-a_{0}\right)} \Delta^{n} \eta
$$

Thus,

$$
\begin{aligned}
\left\|x_{\alpha, m+n}-x_{\alpha, m}\right\| & \leq\left\|x_{\alpha, m+n}-x_{\alpha, m+n-1}\right\|+\cdots+\left\|x_{\alpha, m+1}-x_{\alpha, m}\right\| \\
& \leq \frac{2-a_{0}+(\alpha-1) a_{0}^{2}}{2\left(1-a_{0}\right)}\left[\Delta^{m+n-1}+\cdots+\Delta^{m}\right] \eta \\
& =\frac{2-a_{0}+(\alpha-1) a_{0}^{2}}{2\left(1-a_{0}\right)} \Delta^{m}\left(\frac{1-\Delta^{n}}{1-\Delta}\right) \eta
\end{aligned}
$$

For $m=0$, this gives $x_{\alpha, n} \in \overline{\mathscr{B}}\left(x_{\alpha, 0}, R \eta\right)$. Similarly, it can be proved that $y_{\alpha, n} \in$ $\overline{\mathscr{B}}\left(x_{\alpha, 0}, R \eta\right)$. Hence, $\left\{x_{\alpha, n}\right\}$ is a Cauchy sequence from $\Delta=1 / f\left(a_{0}\right)<1$ and (27). Let $0<a_{0}<r_{0}$ and $b_{0}<\Phi\left(a_{0}\right)$. Now, from (16) and Lemma 3(iii) for $n \geq 1$, we get

$$
\begin{aligned}
\left\|y_{\alpha, n}-x_{\alpha, n}\right\| & \leq c_{n-1}\left\|y_{\alpha, n-1}-x_{\alpha, n-1}\right\| \\
& \leq \cdots \leq\left\|y_{\alpha, 0}-x_{\alpha, 0}\right\| \prod_{j=0}^{n-1} c_{j}<\prod_{j=0}^{n-1}\left(\gamma^{2^{j}} \Delta\right) \eta=\gamma^{2^{n}-1} \Delta^{n} \eta,
\end{aligned}
$$

where, $\gamma=a_{1} / a_{0}<1$ and $\Delta=1 / f\left(a_{0}\right)<1$. Hence, 


$$
\begin{aligned}
&\left\|x_{\alpha, m+n}-x_{\alpha, m}\right\| \\
& \leq\left\|x_{\alpha, m+n}-x_{\alpha, m+n-1}\right\|+\cdots+\left\|x_{\alpha, m+1}-x_{\alpha, m}\right\| \\
& \leq \frac{2-a_{m+n-1}+(\alpha-1) a_{m+n-1}^{2}}{2\left(1-a_{m+n-1}\right)}\left\|y_{\alpha, m+n-1}-x_{\alpha, m+n-1}\right\| \\
&+\cdots+\frac{2-a_{m}+(\alpha-1) a_{m}^{2}}{2\left(1-a_{m}\right)}\left\|y_{\alpha, m}-x_{\alpha, m}\right\| \\
&+\frac{2-a_{m+n-1}+(\alpha-1) a_{m+n-1}^{2}}{2\left(1-a_{m+n-1}\right)} \gamma^{2^{m+n-1}-1} \Delta^{m+n-1} \eta \\
&+\cdots+\frac{2-a_{m}+(\alpha-1) a_{m}^{2}}{2\left(1-a_{m}\right)} \gamma^{2^{m}-1} \Delta^{m} \eta \\
&<\left(\frac{2-a_{m}+(\alpha-1) a_{m}^{2}}{2\left(1-a_{m}\right)}\right) \Delta^{m}\left[\gamma^{2^{m+n-1}-1} \Delta^{n-1}+\cdots+\gamma^{2^{m}-1}\right] \eta \\
&<\left(\frac{2-a_{m}+(\alpha-1) a_{m}^{2}}{2\left(1-a_{m}\right)}\right) \gamma^{2^{m}-1} \Delta^{m}\left[\gamma^{2^{m}\left[2^{n-1}-1\right]} \Delta^{n-1}+\cdots+\gamma^{2^{m}[2-1]} \Delta+1\right] \eta
\end{aligned}
$$

By Bernoulli's inequality, for every real number $x>-1$ and every integer $k \geq 0$, we have $(1+x)^{k}-1 \geq k x$. Thus,

$$
\left\|x_{\alpha, m+n}-x_{\alpha, m}\right\|<\left(\frac{2-a_{0} \gamma^{2^{m}-1}+(\alpha-1) a_{0}^{2} \gamma^{2^{m}-1}}{2\left(1-a_{0} \gamma^{2^{m}-1}\right)}\right) \gamma^{2^{m}-1} \Delta^{m} \frac{1-\gamma^{2^{m} n} \Delta^{n}}{\left(1-\gamma^{2^{m}} \Delta\right)} \eta
$$

For $m=0$, we obtain

$$
\left\|x_{\alpha, n}-x_{\alpha, 0}\right\|<\frac{2-a_{0}+(\alpha-1) a_{0}^{2}}{2\left(1-a_{0}\right)} \frac{1-\gamma^{n} \Delta^{n}}{1-\gamma \Delta} \eta<R \eta
$$

Hence $x_{\alpha, n} \in \mathscr{B}\left(x_{\alpha, 0}, R \eta\right)$. Also $y_{\alpha, n} \in \mathscr{B}\left(x_{\alpha, 0}, R \eta\right)$, is evident from

$$
\begin{aligned}
\left\|y_{\alpha, n+1}-x_{\alpha, 0}\right\| \leq & \left\|y_{\alpha, n+1}-x_{\alpha, n+1}\right\|+\left\|x_{\alpha, n+1}-x_{\alpha, n}\right\|+\cdots+\left\|x_{\alpha, 1}-x_{\alpha, 0}\right\| \\
\leq & \left\|y_{\alpha, n+1}-x_{\alpha, n+1}\right\|+\frac{2-a_{n}+(\alpha-1) a_{n}^{2}}{2\left(1-a_{n}\right)}\left\|y_{\alpha, n}-x_{\alpha, n}\right\| \\
& +\cdots+\frac{2-a_{0}+(\alpha-1) a_{0}^{2}}{2\left(1-a_{0}\right)}\left\|y_{\alpha, 0}-x_{\alpha, 0}\right\| \\
< & \frac{2-a_{n+1}+(\alpha-1) a_{n+1}^{2}}{2\left(1-a_{n+1}\right)}\left\|y_{\alpha, n+1}-x_{\alpha, n+1}\right\| \\
& +\cdots+\frac{2-a_{0}+(\alpha-1) a_{0}^{2}}{2\left(1-a_{0}\right)}\left\|y_{\alpha, 0}-x_{\alpha, 0}\right\| \\
< & \cdots<\frac{2-a_{0}+(\alpha-1) a_{0}^{2}}{2\left(1-a_{0}\right)} \frac{1-\gamma^{n+1} \Delta^{n+1}}{1-\gamma \Delta} \eta<R \eta
\end{aligned}
$$


Taking limit as $n \rightarrow \infty$ in (27) and (29), we get $x^{*} \in \overline{\mathscr{B}}\left(x_{\alpha, 0}, R \eta\right)$. Now we have to show that $x^{*}$ is a solution of $F(x)=0$. We have $\left\|F\left(x_{\alpha, n}\right)\right\| \leq$ $\left\|F^{\prime}\left(x_{\alpha, n}\right)\right\|\left\|\Gamma_{\alpha, n} F\left(x_{\alpha, n}\right)\right\|$ and the sequence $\left\{\left\|F^{\prime}\left(x_{\alpha, n}\right)\right\|\right\}$ is bounded as

$$
\left\|F^{\prime}\left(x_{\alpha, n}\right)\right\| \leq\left\|F^{\prime}\left(x_{\alpha, 0}\right)\right\|+M\left\|x_{n}-x_{0}\right\|<\left\|F^{\prime}\left(x_{0}\right)\right\|+M R \eta .
$$

Now taking limit as $n \rightarrow \infty$, we get $F\left(x^{*}\right)=0$ as $F$ is continuous. To show the uniqueness of the $x^{*}$, let us consider $y^{*}$ be another root of (1) in $\mathscr{B}\left(x_{\alpha, 0}, 2 / M \beta-R \eta\right) \cap \Omega$. Then

$$
0=F\left(y^{*}\right)-F\left(x^{*}\right)=\int_{0}^{1} F^{\prime}\left(x^{*}+t\left(y^{*}-x^{*}\right)\right) d t\left(y^{*}-x^{*}\right)
$$

We have $y^{*}=x^{*}$, if the operator $P=\int_{0}^{1} F^{\prime}\left(x^{*}+t\left(y^{*}-x^{*}\right)\right) d t$ is invertible. From

$$
\begin{aligned}
\left\|I-\Gamma_{0} P\right\|=\left\|\Gamma_{0}\left(F^{\prime}\left(x_{0}\right)-P\right)\right\| & =\left\|\Gamma_{0} \int_{0}^{1}\left[F^{\prime}\left(x^{*}+t\left(y^{*}-x^{*}\right)\right)-F^{\prime}\left(x_{0}\right)\right] d t\right\| \\
& \leq M \beta \int_{0}^{1}\left\|x^{*}+t\left(y^{*}-x^{*}\right)-x_{0}\right\| d t \\
& \leq M \beta \int_{0}^{1}\left((1-t)\left\|x^{*}-x_{0}\right\|+t\left\|y^{*}-x_{0}\right\|\right) d t \\
& <\frac{M \beta}{2}\left(R \eta+\frac{2}{M \beta}-R \eta\right)=1,
\end{aligned}
$$

and by Banach's theorem [10], $P$ is invertible.

\subsection{On $(2+p) R$-order convergence of the method}

We shall establish the $R$-order convergence of the method equals to $(2+p)$ as the method is itself of third-order. For $h(t)=t^{p}, p \in(0,1]$, we get from (14)

$$
A=\int_{0}^{1} h(t)(1-t) d t=\frac{1}{(1+p)(2+p)}
$$

Hence, from (11), the sequence $\left\{b_{n}\right\}$ satisfies

$$
b_{n+1}=b_{n} f\left(a_{n}\right)^{2+p} g\left(a_{n}, b_{n}\right)^{1+p}
$$

with

$$
\begin{aligned}
g(x, y)= & \frac{\alpha x^{2}}{2(1-x)}+\frac{y(1+(\alpha-1) x)}{(1-x)(p+1)(p+2)}+\frac{x^{2}(1+(\alpha-1) x)}{2(1-x)} \\
& +\frac{x^{3}(1+(\alpha-1) x)^{2}}{8(1-x)^{2}} .
\end{aligned}
$$

This gives $g\left(\delta x, \delta^{p+1} y\right)<\delta^{p+1} g(x, y)$, for $x \in\left(0, r_{0}\right]$ and $\delta \in(0,1)$. 
Lemma 4. Let $0<a_{0}<r_{0}, 0<b_{0}<\Phi_{p}\left(a_{0}\right), h(t) \leq t^{p} \leq 1$ and $\gamma=a_{1} / a_{0}$. For $n \geq 1$ we get

(i) $a_{n} \leq \gamma^{(2+p)^{n-1}} a_{n-1} \leq \gamma^{\left((2+p)^{n}-1\right) /(1+p)} a_{0}$ for $n \geq 2$

(ii) $b_{n} \leq\left(\gamma^{(2+p)^{n-1}}\right)^{1+p} b_{n-1} \leq \gamma^{(2+p)^{n}-1} b_{0}$ for $n \geq 2$

(iii) $c_{n} \leq \frac{\gamma^{(2+p)^{n}}}{f\left(a_{0}\right)}, n \geq 0$

Proof. We can prove (i) and (ii) by using induction. Since $a_{1}=\gamma a_{0}$ and $a_{1}<a_{0}$, we get $\gamma<1$. Using (i) of Lemma 1 , we get,

$$
b_{1}=b_{0} f\left(a_{0}\right)^{p+2} g\left(a_{0}, b_{0}\right)^{p+1}<\left(f\left(a_{0}\right)^{2} g\left(a_{0}, b_{0}\right)\right)^{1+p} b_{0}=\left(\frac{a_{1}}{a_{0}}\right)^{1+p} b_{0}=\gamma^{1+p} b_{0}
$$

Thus, (i) and (ii) hold for $n=1$. Suppose (i) and (ii) hold for some $n=k$, then

$$
\begin{aligned}
a_{k+1} & =a_{k} f\left(a_{k}\right)^{2} g\left(a_{k}, b_{k}\right) \leq \gamma^{(p+2)^{k-1}} a_{k-1} f\left(a_{k-1}\right)^{2} g\left(\gamma^{(2+p)^{k-1}} a_{k-1},\left(\gamma^{(2+p)^{k-1}}\right)^{1+p} b_{k-1}\right) \\
& \leq \gamma^{(p+2)^{k-1}} a_{k-1} f\left(a_{k-1}\right)^{2}\left(\gamma^{(2+p)^{k-1}}\right)^{1+p} g\left(a_{k-1}, b_{k-1}\right)=\gamma^{(2+p)^{k}} a_{k}
\end{aligned}
$$

Hence,

$$
\begin{aligned}
a_{k+1} & \leq \gamma^{(2+p)^{k}} a_{k} \leq \gamma^{(2+p)^{k}} \gamma^{(2+p)^{k-1}} a_{k-1} \leq \gamma^{(2+p)^{k}} \gamma^{(2+p)^{k-1}} \cdots \gamma^{(2+p)^{0}} a_{0} \\
& =\gamma^{\left((p+2)^{k+1}-1\right) /(p+1)} a_{0}
\end{aligned}
$$

From $f(x)>1$ in $\left(0, r_{0}\right]$, we get

$$
\begin{aligned}
b_{k+1} & =b_{k} f\left(a_{k}\right)^{p+2} g\left(a_{k}, b_{k}\right)^{p+1} \leq b_{k}\left(f\left(a_{k}\right)^{2} g\left(a_{k}, b_{k}\right)\right)^{p+1} \\
& =b_{k}\left(\frac{a_{k+1}}{a_{k}}\right)^{p+1} \leq\left(\gamma^{(p+2)^{k}}\right)^{p+1} b_{k}
\end{aligned}
$$

Hence,

$$
b_{k+1}=\left(\gamma^{(p+2)^{k}}\right)^{p+1} b_{k}<\left(\gamma^{(p+2)^{k}}\right)^{p+1}\left(\gamma^{(p+2)^{k-1}}\right)^{p+1} \cdots\left(\gamma^{(p+2)^{0}}\right)^{p+1} b_{0}=\gamma^{(p+2)^{k+1}-1} b_{0}
$$

Thus, (i) and (ii) hold for $n \geq 1$. (iii) follows from

$$
\begin{aligned}
c_{n}=f\left(a_{n}\right) g\left(a_{n}, b_{n}\right) & \leq f\left(\gamma^{\left((p+2)^{n}-1\right) /(p+1)} a_{0}\right) g\left(\gamma^{\left((p+2)^{n}-1\right) /(p+1)} a_{0}, \gamma^{(p+2)^{n}-1} b_{0}\right) \\
& \leq \gamma^{(p+2)^{n}} \frac{f\left(a_{0}\right) g\left(a_{0}, b_{0}\right)}{\gamma}=\gamma^{(p+2)^{n}} / f\left(a_{0}\right)
\end{aligned}
$$

as $\gamma=a_{1} / a_{0}=f\left(a_{0}\right)^{2} g\left(a_{0}, b_{0}\right)$. Now,

$$
\left\|y_{\alpha, n}-x_{\alpha, n}\right\| \leq\left(\prod_{j=0}^{n-1} f\left(a_{j}\right) g\left(a_{j}, b_{j}\right)\right) \eta \leq \prod_{j=0}^{n-1}\left(\gamma^{(2+p)^{j}} \Delta\right) \eta=\gamma^{\left((2+p)^{n}-1\right) /(1+p)} \Delta^{n}
$$

and 


$$
\begin{aligned}
\| x_{\alpha, m+n} & -x_{\alpha, m} \| \\
\leq & \frac{2-a_{m}+(\alpha-1) a_{m}^{2}}{2\left(1-a_{m}\right)}\left[\prod_{j=0}^{m+n-2} f\left(a_{j}\right) g\left(a_{j}, b_{j}\right)+\cdots+\prod_{j=0}^{m-1} f\left(a_{j}\right) g\left(a_{j}, b_{j}\right)\right] \eta \\
< & \frac{2-a_{m}+(\alpha-1) a_{m}^{2}}{2\left(1-a_{m}\right)}\left[\gamma^{\left((2+p)^{m+n-1}-1\right) /(1+p)} \Delta^{m+n-1}+\cdots+\gamma^{\left((2+p)^{m}-1\right) /(1+p)} \Delta^{m}\right] \\
< & \frac{\left(2-a_{m}+(\alpha-1) a_{m}^{2}\right) \Delta^{m}}{2\left(1-a_{m}\right)}\left[\gamma^{\left((2+p)^{m+n-1}-1\right) /(1+p)} \Delta^{n-1}+\cdots+\gamma^{\left((2+p)^{m}-1\right) /(1+p)}\right] \eta \\
< & \frac{\left(2-a_{m} \gamma^{\left((2+p)^{m}-1\right) /(1+p)}+(\alpha-1) a_{m}^{2} \gamma^{\left((2+p)^{m}-1\right) /(1+p)}\right) \Delta^{m}}{2\left(1-a_{m} \gamma^{\left((2+p)^{m}-1\right) /(1+p)}\right)} \\
& \times \gamma^{\left((2+p)^{m}-1\right) /(1+p)}\left[\gamma^{(2+p)^{m}\left[(2+p)^{n-1}-1\right] /(1+p)} \Delta^{n-1}\right. \\
& \left.+\cdots+\gamma^{(2+p)^{m}[(2+p)-1] /(1+p)} \Delta^{+} 1\right] \eta
\end{aligned}
$$

By Bernoulli's inequality, we get

$$
\begin{aligned}
\left\|x_{\alpha, m+n}-x_{\alpha, m}\right\|< & \frac{\left(2-a_{m} \gamma^{\left((2+p)^{m}-1\right) /(1+p)}+(\alpha-1) a_{m}^{2} \gamma^{\left((2+p)^{m}-1\right) /(1+p)}\right) \Delta^{m}}{2\left(1-a_{m} \gamma^{\left((2+p)^{m}-1\right) /(1+p)}\right)} \\
& \times\left(\frac{1-\gamma^{(2+p)^{m} n} \Delta^{n}}{1-\gamma^{(2+p)^{m}} \Delta}\right) \eta
\end{aligned}
$$

Now, a priori error bounds for $\left\{x_{\alpha, n}\right\}$ are given by

$$
\begin{aligned}
\left\|x^{*}-x_{\alpha, n}\right\| \leq & \frac{\left(2-\gamma^{\left((2+p)^{n}-1\right) /(1+p)} a_{0}+(\alpha-1) \gamma^{\left((2+p)^{n}-1\right) /(1+p)} a_{0}^{2}\right)}{2\left(1-\gamma^{\left((2+p)^{n}-1\right) /(1+p)} a_{0}\right)} \\
& \times \frac{\gamma^{\left((2+p)^{n}-1\right) /(1+p)} \Delta^{n} \eta}{1-\gamma^{(2+p)^{n}} \Delta}
\end{aligned}
$$

\section{Numerical examples}

In this section, two numerical examples are worked out for demonstrating the efficacy of the continuation method used to solve (1).

Example 1. Let $X=C[a, b]$ be the space of continuous functions on $[a, b]$ and consider the problem of finding the solutions of nonlinear integral equations $F(x)=0$ of mixed type [5], given by

$$
F(x)(s)=x(s)-f(s)-\lambda \int_{a}^{b} G(s, t)\left[x(t)^{2+p}+x(t)^{3}\right] d t, \quad p \in(0,1], \lambda \in \mathbf{R}
$$

where $f, x$ are continuous functions such that $f(s)>0, s \in[a, b]$, and the Kernel $G$ is continuous and nonnegative in $[a, b] \times[a, b]$. 
Solution. Using norm as sup-norm and $G(s, t)$ as the Green's function

$$
G(s, t)= \begin{cases}(b-s)(t-a) /(b-a), & t \leq s, \\ (s-a)(b-t) /(b-a), & s \leq t,\end{cases}
$$

we compute the scalars $M, \beta, \eta$ and the function $\omega(x)$. The first and second derivatives of $F$ can easily be obtained and given by

$$
\begin{gathered}
F^{\prime}(x) u(s)=u(s)-\lambda \int_{a}^{b} G(s, t)\left[(2+p) x(t)^{1+p}+3 x(t)^{2}\right] u(t) d t, \quad u \in \Omega \\
F^{\prime \prime}(x)(u v)(s)=-\lambda \int_{a}^{b} G(s, t)\left[(1+p)(2+p) x(t)^{p}+6 x(t)\right](u v)(t) d t, \quad u, v \in \Omega
\end{gathered}
$$

For $p \in(0,1)$, we must note here that the second derivative $F^{\prime \prime}$ does not satisfy the Lipschitz/Hölder continuity conditions as

$$
\begin{aligned}
& \left\|F^{\prime \prime}(x)-F^{\prime \prime}(y)\right\| \\
& =\left\|\lambda \int_{a}^{b} G(s, t)\left[(1+p)(2+p)\left(x(t)^{p}-y(t)^{p}\right)+6(x(t)-y(t))\right] d t\right\| \\
& \quad \leq|\lambda| \max _{s \in[a, b] \mid}\left|\int_{a}^{b} G(s, t) d t\right|\left[(1+p)(2+p)\left\|x(t)^{p}-y(t)^{p}\right\|+6\|x(t)-y(t)\|\right] \\
& \quad \leq|\lambda|\|l\|\left[(1+p)(2+p)\|x-y\|^{p}+6\|x-y\|\right], \quad \forall x, y \in \Omega,
\end{aligned}
$$

where

$$
\|l\|=\max _{s \in[a, b]}\left|\int_{a}^{b} G(s, t) d t\right|
$$

However, it satisfies the $\omega$-continuity condition given by

$$
\left\|F^{\prime \prime}(x)-F^{\prime \prime}(y)\right\| \leq \omega(\|x-y\|), \quad \forall x, y \in \Omega,
$$

where, $\omega(x)=|\lambda|\|l\|\left[(1+p)(2+p) x^{p}+6 x\right]$. This leads to $\omega(t x) \leq t^{p} \omega(x)$, for $p \in(0,1)$ and $t \in[0,1]$. Hence, $h(t)=t^{p}$, and $K=\int_{0}^{1} h(t)(1-t) d t+\frac{1}{2} \int_{0}^{1} h(t) d t$ $=\frac{p+4}{2(p+1)(p+2)}$. It is easy to compute

$$
\left\|F\left(x_{\alpha, 0}\right)\right\| \leq\left\|x_{\alpha, 0}-f\right\|+|\lambda|\|l\|\left[\left\|x_{\alpha, 0}\right\|^{2+p}+\left\|x_{\alpha, 0}\right\|^{3}\right]
$$

and

$$
\left\|F^{\prime \prime}(x)\right\| \leq|\lambda|\|l\|\left[(1+p)(2+p)\|x\|^{p}+6\|x\|\right]
$$

This gives $M=|\lambda|\|l\|\left[(1+p)(2+p)\|x\|^{p}+6\|x\|\right]$. Also

$$
\left\|I-F^{\prime}\left(x_{\alpha, 0}\right)\right\| \leq|\lambda|\|l\|\left[(2+p)\left\|x_{\alpha, 0}\right\|^{1+p}+3\left\|x_{\alpha, 0}\right\|^{2}\right]
$$



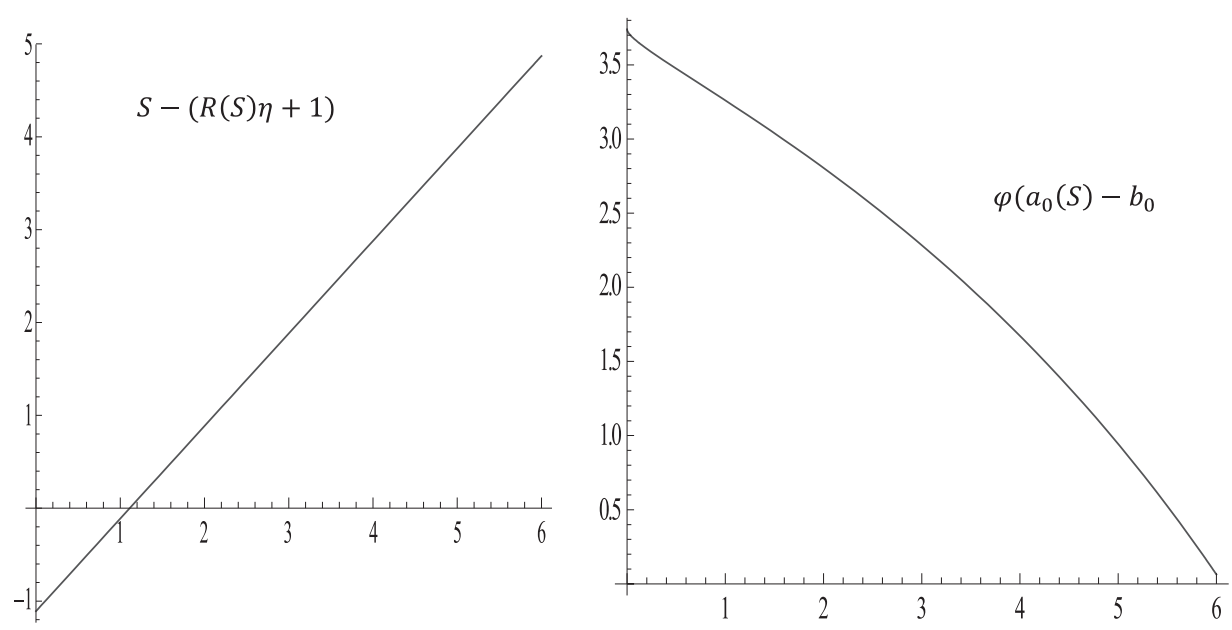

FIGURE 1. Conditions on the parameter $S$ for $\alpha=0$

Now, if $|\lambda|\|l\|\left[(2+p)\left\|x_{\alpha, 0}\right\|^{1+p}+3\left\|x_{\alpha, 0}\right\|^{2}\right]<1$, then by Banach's theorem [10], we obtain

$$
\left\|\Gamma_{\alpha, 0}\right\|=\left\|F^{\prime}\left(x_{\alpha, 0}\right)^{-1}\right\| \leq \frac{1}{1-|\lambda|\|l\|\left[(2+p)\left\|x_{\alpha, 0}\right\|^{1+p}+3\left\|x_{\alpha, 0}\right\|^{2}\right]}=\beta
$$

and

$$
\left\|\Gamma_{\alpha, 0} F\left(x_{\alpha, 0}\right)\right\| \leq \frac{\left\|x_{\alpha, 0}-f\right\|+|\lambda|\|l\|\left[\left\|x_{\alpha, 0}\right\|^{2+p}+\left\|x_{\alpha, 0}\right\|^{3}\right]}{1-|\lambda|\|l\|\left[(2+p)\left\|x_{\alpha, 0}\right\|^{1+p}+3\left\|x_{\alpha, 0}\right\|^{2}\right]}=\eta
$$

For $a=0$ and $b=1$, we get

$$
\|l\|=\max _{s \in[0,1]}\left|\int_{0}^{1} G(s, t) d t\right|=1 / 8
$$

For $\lambda=1 / 3, p=1 / 2, f(s)=1$, and initial point $x_{\alpha, 0}=x_{0}(s)=1$ in $[0,1]$, we get $\left\|\Gamma_{\alpha, 0}\right\| \leq \beta=1.2973,\left\|\Gamma_{\alpha, 0} F\left(x_{\alpha, 0}\right)\right\| \leq \eta=0.108108, \omega(\eta)=0.0784017$ and $b_{0}=\beta \eta \omega(\eta)=0.0109957$. The conditions of Theorem 1 requires to find the values of a parameter $S$ such that $S \in \overline{\mathscr{B}}\left(x_{\alpha, 0}, R \eta\right) \subseteq \Omega$. The values of $S \in$ $(1.11205,6.06526)$ are obtained from Figure 1 for $\alpha=0$ such that $S-(R(S) \eta+1)>0$ and $\Phi\left(a_{0}(S)\right)-b_{0} \leq 0$. Also, $a_{0}(S) \leq r_{0}=0.380778$ if and only if $S<8.98651$. Hence, if we choose $S=5$, then we have $\Omega=\mathscr{B}(1,5), \quad M=1.59939, a_{0}=0.224311$ and $b_{0}=0.0109957<\Phi(0.224311)$. Thus, the conditions of the Theorem 1 are satisfied. Hence, a solution of equation (34) exists in the ball $\overline{\mathscr{B}}(1,0.128752) \subseteq \Omega$ and unique in the ball $\mathscr{B}(1,0.950051) \cap \Omega$. Now, for $\alpha=1$ we get $S \in(1.11205,6.06256)$ from Figure 2. Again for $S=5$, we get $a_{0}=0.321311<0.380778$ and $b_{0}<\Phi\left(a_{0}\right)$ and a 

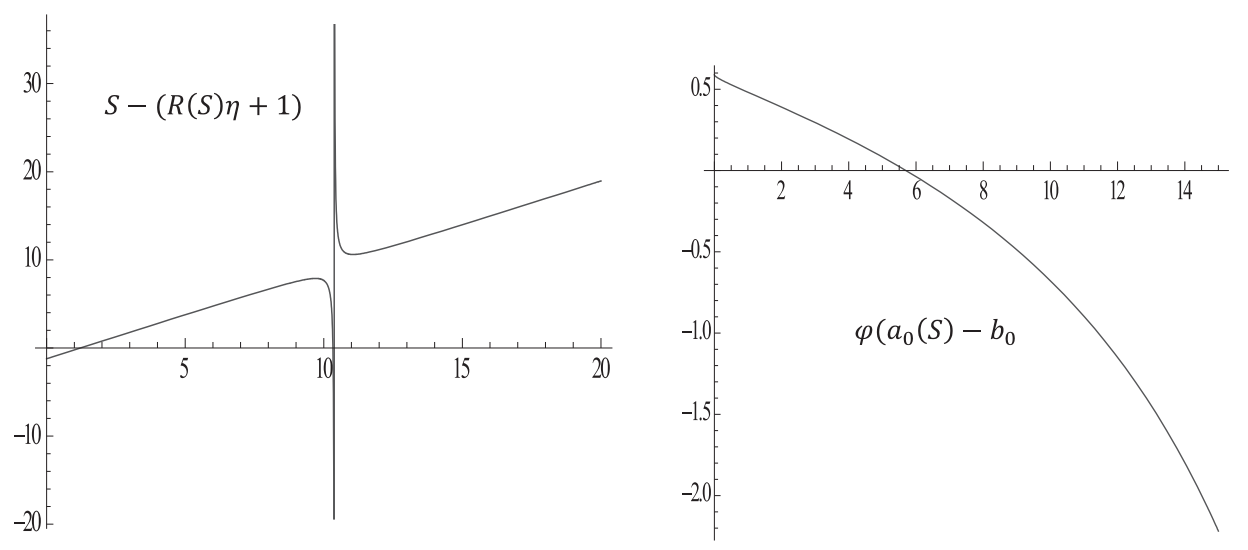

Figure 2. Conditions on the parameter $S$ for $\alpha=1$

solution of (34) exists in $\overline{\mathscr{B}}(1,0.141648) \subseteq \Omega$ and is unique in the ball $\mathscr{B}(1,0.948604) \cap \Omega$. On the other hand from [4], we get $A$ and $\Psi(x)$ as $A=$ $\int_{0}^{1}(1-t) t^{p}=\frac{1}{(1+p)(2+p)}, \Psi(x)=\frac{2\left(x^{2}-3 x+1\right)}{A(2-x)}$. Now, to get $S$ from Theorem 2.6 [4], it is necessary that $\overline{\mathscr{B}}\left(x_{0}, R \eta\right) \subseteq \Omega$. For this, it is sufficient to check $S-(R(S) \eta+1)>0$ and $\Psi\left(a_{0}(S)\right)-b_{0}>0$. This gives $S \in(1.4689,11.9076)$ as is evident from Figure 3. Also, $a_{0}(S)<(3-\sqrt{5}) / 2$ if and only if $S<9.0172$. Hence, if we choose $S=5$ then we get $\Omega=\mathscr{B}(1,5), M=1.599385, a_{0}=0.22431$ and $b_{0}=0.0109957<\Psi\left(a_{0}\right)$. Thus, in this case also, the conditions of the Theorem 1 are satisfied. Hence, a solution of (34) exists in the ball $\overline{\mathscr{B}}(1,0.12862) \subseteq \Omega$ and unique in the ball $\mathscr{B}(1,0.8529) \cap \Omega$. From this, we observe that our convergence analysis gives better existence and uniqueness ball than that of [4].
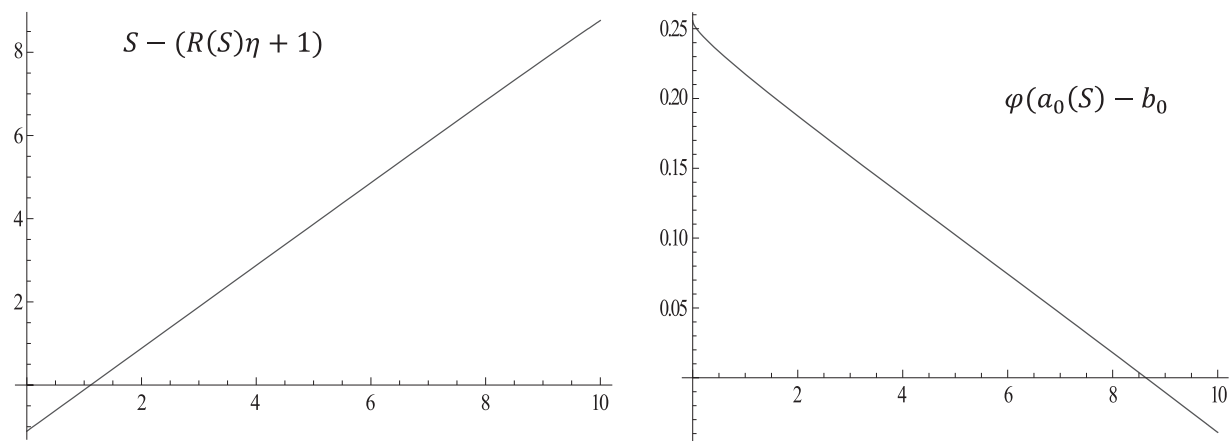

FIGURE 3. Conditions on the parameter $S$ 
Example 2. Let $X=C[0,1]$ be the space of continuous functions on $[0,1]$ and let us consider the integral equations $F(x)=0$ on $\mathbf{X}$, where

$$
F(x)(s)=x(s)-f(s)-\lambda \int_{0}^{1} \frac{s}{s+t} x(t)^{2+p} d t,
$$

with $s \in[0,1], x, f \in X, p \in(0,1)$ and $\lambda$ is a real number. These types of integral equations are known as Fredholm type (cf. Davis [3]).

Solution. Here, norm is taken as the sup-norm. Now, it is easy to find the first and second Fréchet derivatives of $F$ as

$$
\begin{gathered}
F^{\prime}(x) u(s)=u(s)-\lambda(2+p) \int_{0}^{1} \frac{s}{s+t} x(t)^{1+p} u(t) d t, \quad u \in \Omega \\
F^{\prime \prime}(x)(u v)(s)=-\lambda(1+p)(2+p) \int_{0}^{1} \frac{s}{s+t} x(t)^{p}(u v)(t) d t, \quad u, v \in \Omega
\end{gathered}
$$

Clearly, $F^{\prime \prime}$ does not satisfies the Lipschitz continuity condition as for $p \in(0,1)$ and for all $x, y \in \Omega$

$$
\begin{aligned}
\left\|F^{\prime \prime}(x)-F^{\prime \prime}(y)\right\| & =\left\|\lambda(2+p)(1+p) \int_{0}^{1} \frac{s}{s+t}\left[x(t)^{p}-y(t)^{p}\right] d t\right\| \\
& \leq|\lambda|(2+p)(1+p) \max _{s \in[0,1]\left|\int_{0}^{1} \frac{s}{s+t} d t\right|\left\|x(t)^{p}-y(t)^{p}\right\|} \\
& \leq|\lambda|(2+p)(1+p) \log 2\|x-y\|^{p}
\end{aligned}
$$

However, it satisfies the Hölder continuity condition for $p \in(0,1]$ and $N=$ $|\lambda|(2+p)(1+p) \log 2$. To obtain a bound for $\Gamma_{\alpha, 0}$, we find

$$
\left\|F\left(x_{\alpha, 0}\right)\right\| \leq\left\|x_{\alpha, 0}-f\right\|+|\lambda| \log 2\left\|x_{\alpha, 0}\right\|^{p}
$$

and

$$
\left\|I-F^{\prime}\left(x_{\alpha, 0}\right)\right\| \leq|\lambda|(2+p) \log 2\left\|x_{\alpha, 0}\right\|^{1+p}
$$

Now, if $|\lambda|(2+p) \log 2\left\|x_{\alpha, 0}\right\|^{1+p}<1$, then by Banach Lemma, we get

$$
\left\|\Gamma_{\alpha, 0}\right\|=\left\|F^{\prime}\left(x_{\alpha, 0}\right)^{-1}\right\| \leq \frac{1}{1-|\lambda|(2+p) \log 2\left\|x_{\alpha, 0}\right\|^{1+p}}=\beta
$$

Also,

$$
\left\|F^{\prime \prime}(x)\right\| \leq|\lambda|(2+p)(1+p) \log 2\|x\|^{p}
$$

Hence,

$$
\left\|\Gamma_{\alpha, 0} F\left(x_{\alpha, 0}\right)\right\| \leq \frac{\left\|x_{\alpha, 0}-f\right\|+|\lambda| \log 2\left\|x_{\alpha, 0}\right\|^{p}}{1-|\lambda|(2+p) \log 2\left\|x_{\alpha, 0}\right\|^{1+p}}
$$


Now, for $\lambda=1 / 10, p=1 / 2, f(s)=1$, and initial point $x_{0}=x_{0}(s)=1$ in the interval $[0,1]$, we get $\left\|\Gamma_{\alpha, 0}\right\| \leq \beta=1.20961,\left\|\Gamma_{0} F\left(x_{0}\right)\right\| \leq \eta=0.0838437, \quad N=$ 0.25993 and $b_{0}=\beta \eta \omega(\eta)=0.00763322$. The conditions of Theorem 1 requires to find the values of a parameter $S$ such that $S \in \overline{\mathscr{B}}\left(x_{\alpha, 0}, R \eta\right) \subseteq \Omega$. The values of $S \in(1.08521,102.403)$ are obtained from Figure 4 for $\alpha=0$ such that $S-(R(S) \eta+1)>0$ and $\Phi\left(a_{0}(S)\right)-b_{0} \leq 0$. Also, $a_{0}(S) \leq r_{0}=0.380778$ if and only if $S<208.651$. From $M=M(S)=0.25993 S^{p}, a_{0}=a_{0}(S)=M(S) \beta \eta$ $=0.0263616 S^{p}$ for $S=90$, we get $\Omega=\mathscr{B}(1,90), M=2.46591, a_{0}=0.250088$, $b_{0}=0.0076332<0.404481=\Phi(0.250088)$. Thus, the conditions of Theorem 1 are satisfied. Hence, a solution of (35) exists in $\overline{\mathscr{B}}(1,0.1091953) \subseteq \Omega$ and is unique in the ball $\mathscr{B}(1,0.662196) \cap \Omega$. Now, for $\alpha=1$, we get $S \in(1.08521,102.403)$ from Figure 5. Again taking $S=90$ then $a_{0}=0.250088<0.380778$ and $b_{0}=$ $0.0076332<0.404481=\Phi(0.250088)$, we find that a solution of (35) exists in $\overline{\mathscr{B}}(1,0.111992) \subseteq \Omega$ and unique in the ball $\mathscr{B}(1,0.661123) \cap \Omega$. On the other hand from [4], we get $A$ and $\Psi(x)$ as $A=\int_{0}^{1}(1-t) t^{p}=\frac{1}{(1+p)(2+p)}, \Psi(x)=$
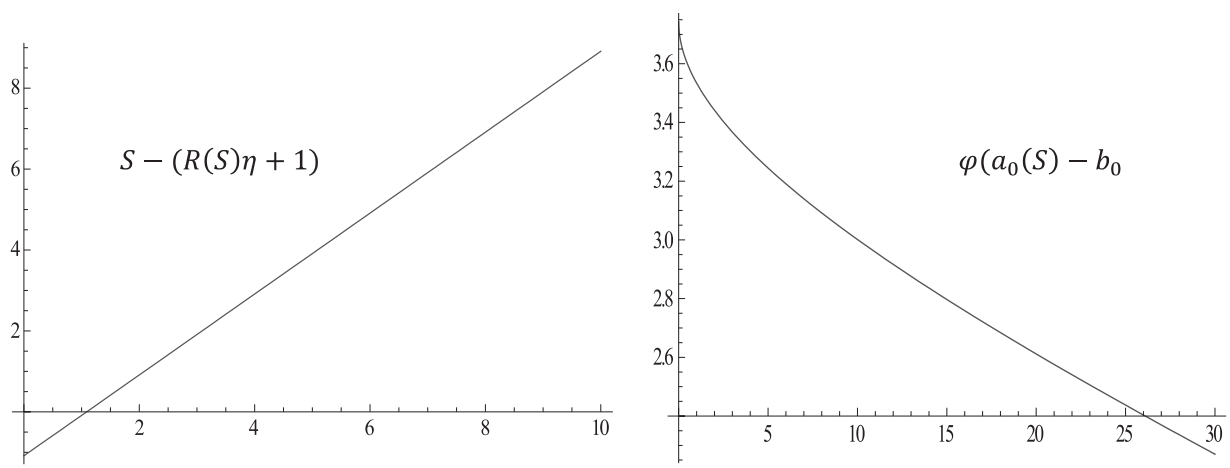

FIGURE 4. Conditions on the parameter $S$ for $\alpha=0$
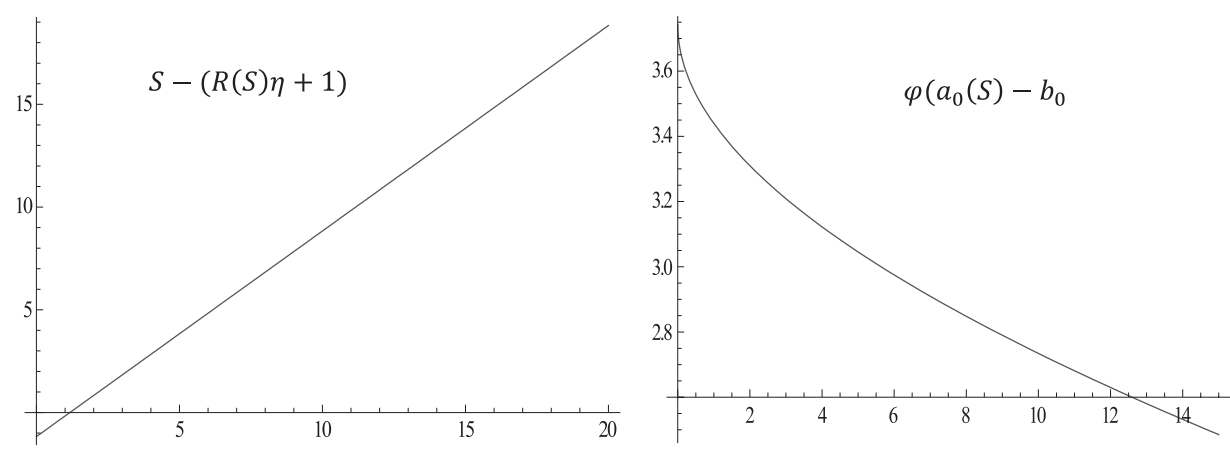

FIGURE 5. Conditions on the parameter $S$ for $\alpha=1$ 

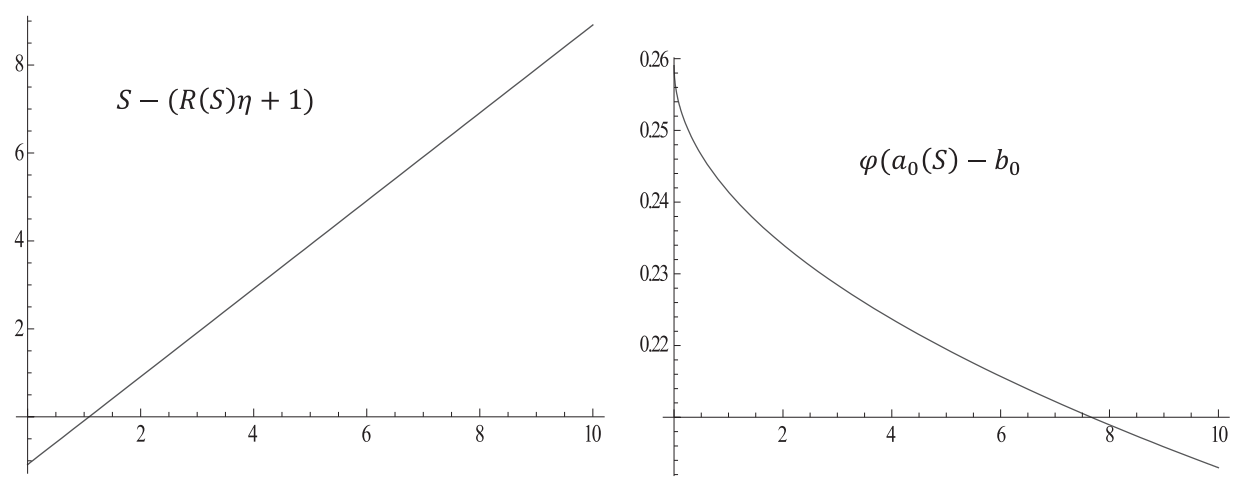

FIGURE 6. Conditions on the parameter $S$

$\frac{2\left(x^{2}-3 x+1\right)}{A(2-x)}$. Now, to get $S$ from Theorem $2.6[4]$, it is necessary that $\overline{\mathscr{B}}\left(x_{0}, R \eta\right) \subseteq \Omega$. For this, it is sufficient to check $S-(R(S) \eta+1)>0$ and $\Psi\left(a_{0}(S)\right)-b_{0}>0$. This gives $S \in(1.08523,198.695)$ as is evident from Figure 6. Also, $a_{0}(S)<(3-\sqrt{5}) / 2$ if and only if $S<209.955$. Hence, if we choose $S=90$ then we get $\Omega=\mathscr{B}(1,90), \quad M=2.46591, a_{0}=0.250088$ and $b_{0}=$ $0.0076332<\Psi\left(a_{0}\right)$. Thus, in this case also, the conditions of the Theorem 1 are satisfied. Hence, a solution of equation (35) exists in the ball $\overline{\mathscr{B}}(1,0.103038)$ $\subseteq \Omega$ and unique in the ball $\mathscr{B}(1,0.661874) \cap \Omega$. From this, we observe that our convergence analysis gives better existence ball but not good uniqueness ball than that of [4].

\section{Conclusions}

In this paper, the semilocal convergence of a parameter based continuation method combining the Chebyshev's and the Super-Halley's methods for solving nonlinear equations in Banach spaces is described. The parameter $\alpha \in[0,1]$ be such that for $\alpha=0$ it reduces to the Chebyshev's method and for $\alpha=1$ to the Super-Halley's method. This convergence is established using recurrence relations under the assumption that the second order Fréchet derivative satisfies the $\omega$-continuity condition. This condition is milder than the Lipschitz and the Hölder continuity conditions used for this purpose. A numerical example is given to show that the second order Fréchet derivative satisfies the $\omega$-continuity condition even when it fails to satisfy the Lipschitz and the Hölder continuity conditions. A number of recurrence relations are derived based on two parameters. An existence-uniqueness theorem is also established to show that the $R$-order convergence of the method is $(2+p), p \in(0,1]$ along with the estimation of a priori error bounds. Two numerical examples are worked out to demonstrate the efficacy of the method. It is observed that our method gives better 
existence and uniqueness regions of the solution for both the examples when compared with the results obtained in [4] for both the Chebyshev's method $(\alpha=0)$ and the Super-Halley's method $(\alpha=1)$.

\section{REFERENCES}

[1] E. L. Allgower And K. Georg, Numerical continuation methods, an introduction, SpringerVerlag, New York, 1990.

[2] V. Candela and A. Marquina, Recurrence relations for rational cubic methods II: The Chebyshev method, Computing 45 (1990), 355-367.

[ 3 ] H. T. DAvis, Introduction to nonlinear differential and integral equations, Dover, New York, 1962.

[ 4 ] J. A. Ezquerro And M. A. Hernández, On the R-order of the Halley method, J. Math. Anal. Appl. 303 (2005), 591-601.

[5] M. Ganesh AND M. C. Joshi, Numerical solvability of Hammerstein integral equations of mixed type, IMA J. Numer. Anal. 11 (1991), 21-31.

[6] D. K. Gupta and M. Prashanth, Semilocal convergence of a continuation method with Hölder continuous second derivative in Banach spaces, J. Compt. Appl. Math. 236 (2012), 3174-3185.

[ 7 ] J. M. GutiérRez AND M. A. Hernández, Recurrence relations for the super-Halley method, Compt. Math. Appl. 36 (1998), 1-8.

[8] M. A. Hernández, Second derivative free variants of Chebyshev method for nonlinear equations, J. Optim. Theory Appl. 104 (2000), 501-515.

[9] M. A. Hernández and M. A. Salanova, Modification of the Kantorovich assumptions for semilocal convergence of the Chebyshev method, J. Compt. Appl. Math. 126 (2000), $131-143$.

[10] L. V. Kantorovich and G. P. Akilov, Functional analysis, Pergamon Press, Oxford, 1982.

[11] D. KincaId AND W. Cheney, Numerical analysis: Mathematics of scientific computing, Brooks/Cole, 1991.

[12] A. M. Ostrowski, Solution of equations and systems of equations, Academic Press Inc, 1966.

[13] P. K. Parida and D. K. Gupta, Semilocal convergence of a family of third order Chebyshevtype methods under a mild differentiability condition, Int. J. of Compt. Math. 87 (2010), 3405-3419.

[14] M. Prashanth AND D. K. GuPta, Recurrence relations for Super-Halley's method with Hölder continuous second derivative in Banach spaces, Kodai Math. J. 36 (2013), 119136.

[15] L. B. RALL, Computational solution of nonlinear operator equations, Wiley, New York, 1969.

[16] X. YE AND C. LI, Convergence of the family of the deformed Euler-Halley iteration under the Hölder condition of the second derivative, J. Compt. Appl. Math. 194 (2006), 294308.

[17] Y. ZhaO AND Q. Wu, Newton-Kantorovich theorem for a family of modified Halley's method under Hölder continuity conditions in Banach space, Appl. Math. Comput. 202 (2008), 243-251. 
Maroju Prashanth

DEPARTMENT OF MATHEMATICS

INDIAN InSTITUTE OF TECHNOLOGY

KHARAGPUR - 721302

INDIA

E-mail: maroju.prashanth@gmail.com

Dharmendra K. Gupta

DEPARTMENT OF MATHEMATICS

Indian Institute of TeChNOLOGY

KHARAGPUR - 721302

INDIA

E-mail: dkg@maths.iitkgp.ernet.in 